www.jmscr.igmpublication.org

Impact Factor 5.84

Index Copernicus Value: 83.27

ISSN (e)-2347-176x ISSN (p) 2455-0450

crossref DOI: _https://dx.doi.org/10.18535/jmscr/v5i6.85

\title{
Study of Serum Hypomagnesemia in Critically Ill Patients Admitted in Medical ICU of Hamidiya Hospital
}

\author{
Authors \\ T.N. Dubey, K K Kawre, Ravi Patel \\ GMC BHOPAL
}

\section{Introduction}

Magnesium is the fourth most abundant cation in human body and the second most intracellular cation after potassium. It is a critical ion that is essential for life. It serves as a cofactor for more than 300 enzymatic reactions mainly involving the transfer of phosphate group, for example formation of ATP. It also has important endocrine functions and is required for protein synthesis. It also maintains neuromuscular excitability and is important for maintenance of cardiac function.

There a multiple reasons for magnesium deficiency in critical care settings example:Decreased absorption caused by impaired gastrointestinal activity, malnutrition, renal wasting due to various drug (Digoxin, Gentamicin, Loop Diuretics), Diabetis mellitus, Hypokalemia and Hypocalcemia. Therefor understanding the causes of Hypomagnesemia may be important to define and improve patient prognosis. Hypomagnesemia is implicated in development of systemic inflammatory response syndrome and sepsis in ICU Patients. Also implicated as a cause of seizures, torsades de points.

It has been estimated that 20 to $65 \%$ of critically ill patients develop hypomagnesemia during the course of their ICU Stays. Hypomagnesemia, thouse so common in critically ill, is Frequently overlooked magnesium depletion is described as the most underdiagnosed abnormality in clinical practice. Potancial relationship between hypomagnasemia and in increase mortality has been suggested in literature.

Many studies have been done previously showing varied prevalence and increased association with mortality and morbidity in these patients. My aim in this study to find out the propotion of Hypomagnesemia in critically ill medical patients and to correlate the serum magnesium levels with patients mortality and morbidity outcome considering the length of ICU Stay, need for veltilatory support, primary medical conditions like Sepsis and diabetes and other electrolyte abnormalities associated with, if any in critically ill patients admitted in out medical intensive care unit.

\section{Aims and Objectives}

1. To find out the proportion of hypomagnesemia in critically ill medical patients.

2. To relate the serum magnesium levels with patients mortality and morbidity outcome considering the length of ICU stay, need for ventilatory supports, primary medical conditions and other electrolyte abnormalities associated with if any in 
critically ill patients admitted to medical intensive care unit.

\section{Material and Methods}

We conducted a prospective observational study in our medical intensive care unit of a tertiary care hospital from 1 march 2015 to 30 September 2016. The study was approved by the Institutional Ethical Committee. Fifty patients admitted to the medical ICU for critical illness with vital instability requiring intensive care for more than at least 2 days with age more than 12 years were included after a written informed consent was obtained. Patients receiving magnesium supplementation prior to transfer to ICU were excluded from this study. A blood sample was collected for estimation of serum total magnesium level on the day of admission to ICU. A detailed history and through clinical examination were performed in every patient. Other biochemical, haemtological Investigations were done by standered laboratory method and also radiological investigations as indicated in every patient. In the day of admission the SOFA score has been calculated. Patients were followed up to assess their mortality and morbidity in the form of total ICU and hospital stay, need of mechanical ventilation and duration of ventilator assistance, associated medical conditions like sepsis, diabetes and other electrolyte abnormalities. The study did not interfere with the patient management in the ICU. The normal value of total serum magnesium was between 1.7 to $2.4 \mathrm{mg} / \mathrm{dl}$. Duration of ventilation was defined as the number of days with mechanical ventilation. Patients were classified into two groups according to their initial serum total magnesium level: huypomagnesemia and normomagnesemia. Finally all collected data were tabulated and analysed using standard statistical methods by SPSS version 20 for windows. Qualitative data were analysed by chisquare test, quantitative data by unpaired student $\mathrm{t}$ test, correlation by Pearson's correlation coefficient test and multivariate regression for individual risk assessments.
Inclusion Criteria - Those patients admitted in ICU (Medicine).

Exclusion Criteria - Patients treated previously with magnesium.

Method of Estimation of Serum Magnesium :

(a) Collection of Sample

(b) Preparation of Sample

(c) Method

\section{(A) Collection of Sample}

$5 \mathrm{ml}$. blood of the patient is collected by venepuncture by disposable syringe and transfered to polyethylene test tubes and kept aside for 1 hour. It is then centrifuged at 2500 r.p.m. The serum is then transferred to plastic bottle by pipette and stored in deep freezer.

\section{(B) Preparation of Sample}

Frozen sample are taken out of the deep freezerr and allowed to attain room temperature.

\section{(C) Method}

The serum magnesium estimation is done by modified titan-yellow method as cited by Hawk. In this method polyvinyl alcohol is used as colour stabilizing and colour intensifying agent. The practical advantage is that small amount of serum is required and there is enhacement of production of colour and avoidance of loss of magnesium on protein precipitation.

\section{Principle}

Magnesium is protein free filtrate which is complexed with titan-yellow in an alkaline medium and resulting red lake is measured by spectrophotometer cistronic type 102.

\section{Reagents}

1. 2/3 N. Sulfuric Acid. Weigh 35 gms. of concentrated sulfuric acid in small beaker, dilute to 1 litre with water and mix well. Check by titration against standard alkali and adjust if necessary.

2. $10 \%$ sodium tungstate solution. Dissolve 100 gms. of reagent grade, carbonate free sodium tungstate in water and dilute to one litre. It is stable indefinitely.

3. Polyvinyl alcohol $0.05 \%$ Dissolve 0.125 gms. of polyvinyl alcohol (low viscosity type) in distilled water by stirring in a 
$65^{\circ} \mathrm{C}$ water bath. Dilute to $250 \mathrm{ml}$. of water and filter. Titan Yellow $0.05 \%$. Dissolve $0.1 \mathrm{gm}$. of Titan Yellow powder in $200 \mathrm{ml}$. of distilled water.

4. N. Sodiumhydroxide. Dissolve 160 gins. of Sodium hydroxide in enough water to make one litre.

5. Calcium chloride solution. Dissolve 16.13 $\mathrm{mg}$. of calcium chloride in enough water to make $100 \mathrm{ml}$.

6. Stock magnesium standard. Dissolve 40.4 gms. of magnesium phosphate in $1 \mathrm{ml}$. hydrochloric acid and make upto one litre.

7. Magnesium standard. Dilute $1 \mathrm{ml}$. of stock standard to $200 \mathrm{ml}$. with distilled water. This solution contains $0.02 \mathrm{mg}$. magnesium $/ \mathrm{ml}$.

\section{Procedure}

(a) Separate the serum from clot as soon as possible to avoid the passage of magnesium from $\mathrm{RBC}$ to serum.

(b) Prepare protein free filtrate by adding $1 \mathrm{ml}$. of non-haemolysed serum to $55 \mathrm{~m} 1$. of water, $2 \mathrm{ml}$. of sodium tungstate and $2 \mathrm{ml}$. of $2-3 \mathrm{~N}$ sulfuric acid.

(c) Mix well and centrifuge at high speed for 5 minutes.

(d) Transfer $5 \mathrm{ml}$. of filtrate to test-tube containing $1 \mathrm{ml}$. of water and $1 \mathrm{ml}$. of polyvinyl alcohol. Mix well.

(e) Add $1 \mathrm{ml}$. of titan yellow and $2 \mathrm{ml}$. of $4 \mathrm{~N}$. sodium hydroxide. Mix well.

(f) Blank reagent is made using $1 \mathrm{ml}$. of calcium chloride solution in place of serum and standard using 1 inl. of working magnesium standard in place of serum. Carry out both through the entire procedure.

(g) Set the spectrophotometer to zero with water. Take reading approximately after 1 minute of adding sodium hydroxide at 540 millimicron.

\section{Observation and Results}

The present study was conducted in the department of medicine hamidia hospital Bhopal from 1 march 2015 to 30 september 2016. total no of case in this study is- 50

Table No. 1 Age Wise Distribution of Patients

\begin{tabular}{|l|c|c|c|c|c|}
\hline S No & Age & $\begin{array}{c}\text { No. of } \\
\text { cases }\end{array}$ & $\begin{array}{c}\text { Normomagnese } \\
\text { mia }\end{array}$ & Hypomagnesaemia & Hypermagnesaemia \\
\hline 1 & $10-20$ & 2 & 1 & 1 & 0 \\
\hline 2 & $21-30$ & 2 & 1 & 1 & 1 \\
\hline 3 & $31-40$ & 6 & 0 & 5 & 3 \\
\hline 4 & $41-50$ & 8 & 6 & 3 & 0 \\
\hline 5 & $51-60$ & 15 & 6 & 10 & 0 \\
\hline 6 & $61-70$ & 11 & 2 & 8 & 0 \\
\hline 7 & $71-80$ & 2 & 1 & 2 & 5 \\
\hline & & Total & 15 & 30 & 1 \\
\hline
\end{tabular}

Table no 1 shows proportion of hypomagnesemia (60\%), hypermagnesemia(10\%), normomagnesemia $(30 \%)$. 
Table No 2 Showing Low Serum Magnesium Level with Serum Potassium Level in Case Of Critically Ill Patients

\begin{tabular}{|l|c|c|c|c|}
\hline s.no. & Case no. & Hypomagnesaemia & S.potassium & pvalue \\
\hline 1 & 6 & 0.8 & 2.3 & 0.05 \\
\hline 2 & 7 & 0.9 & 4.78 & 0.0129 \\
\hline 3 & 9 & 1.4 & 5.2 & 0.1266 \\
\hline 4 & 10 & 1 & 3.2 & 0.3333 \\
\hline 5 & 12 & 1.2 & 4.3 & 0.1033 \\
\hline 6 & 15 & 1.1 & 2.26 & 0.0386 \\
\hline 7 & 16 & 1.2 & 4.4 & 0.1066 \\
\hline 8 & 18 & 1.1 & 4.5 & 0.1133 \\
\hline 9 & 19 & 1 & 4.8 & 0.1266 \\
\hline 10 & 20 & 1.08 & 4.6 & 0.1173 \\
\hline 11 & 21 & 1.03 & 4.3 & 0.1090 \\
\hline 12 & 22 & 1.08 & 3.8 & 0.9066 \\
\hline 13 & 23 & 1.6 & 3.8 & 0.7333 \\
\hline 14 & 24 & 0.9 & 4.1 & 0.1066 \\
\hline 15 & 25 & 0.8 & 4.1 & 0.1106 \\
\hline 16 & 26 & 1.2 & 3.6 & 0.0802 \\
\hline 17 & 27 & 1.6 & 4.1 & 0.8333 \\
\hline 18 & 29 & 0.7 & 3.8 & 0.1303 \\
\hline 19 & 31 & 0.9 & 3.4 & 0.0300 \\
\hline 20 & 32 & 0.9 & 3.4 & 0.0300 \\
\hline 21 & 33 & 0.4 & 4.5 & 0.1500 \\
\hline 22 & 34 & 1.1 & 3.5 & 0.0366 \\
\hline 23 & 35 & 1 & 3.2 & 0.0333 \\
\hline 24 & 36 & 0.9 & 4.78 & 0.1293 \\
\hline 25 & 37 & 1.3 & 3 & 0.0433 \\
\hline 26 & 38 & 1.08 & 4.6 & 0.1173 \\
\hline 27 & 40 & 1.6 & 3.8 & 0.0733 \\
\hline 28 & 41 & 0.3 & 4.1 & 0.1266 \\
\hline 29 & 48 & 0.8 & 2.5 & 0.0266 \\
\hline 30 & 50 & 1.3 & 3.2 & 0.0433 \\
\hline & Mean & 1.04233 & 3.864 & 0.089112 \\
\hline
\end{tabular}

above table shows mean serum magnesium level is 1.04233 in hypomagnesemic patients and mean

s. potassium level was 3.864.and p value is below 0.05 in hypokalemia

Table 3 Showing Normal Magnesium Level With Serum Potassium Level

\begin{tabular}{|l|c|c|c|c|}
\hline s.no & Case no. & Normomagnesemia & Hypokalemia & P value \\
\hline 1 & 2 & 1.8 & 3.8 & 0.0660 \\
\hline 2 & 3 & 1.8 & 4.5 & 0.0902 \\
\hline 3 & 5 & 1.9 & 4.5 & 0.0866 \\
\hline 4 & 8 & 1.7 & 3.2 & 0.0500 \\
\hline 5 & 11 & 1.8 & 4.1 & 0.0766 \\
\hline 6 & 13 & 1.8 & 4.2 & 0.0800 \\
\hline 7 & 14 & 1.8 & 4 & 0.7333 \\
\hline 8 & 28 & 1.8 & 3.8 & 0.0666 \\
\hline 9 & 30 & 1.8 & 3.7 & 0.0633 \\
\hline 10 & 39 & 1.9 & 4.6 & 0.0900 \\
\hline 11 & 42 & 1.9 & 4.6 & 0.0900 \\
\hline 12 & 44 & 1.8 & 4.3 & 0.1166 \\
\hline 13 & 45 & 1.9 & 4.2 & 0.0433 \\
\hline 14 & 47 & 1.8 & 3.7 & 0.0633 \\
\hline 15 & 49 & 1.9 & 2.5 & 0.1200 \\
\hline & Mean & 1.82667 & 3.98 & $0 . .08922$ \\
\hline
\end{tabular}

above table shows mean serum magnesium level is 1.82667 in normomagnesemic patients and mean s. potassium level was 3.98 and maximum patients $\mathrm{p}$ value is greater than 0.05 . 
Table - 4 Showing Low Serum Magnesium Level with Serum Sodium Level in Case 0f Critically Ill Patients

\begin{tabular}{|c|c|c|c|}
\hline s.no. & Case no. & Hypomagnesaemia & Hyponatremia \\
\hline 1 & 6 & 0.8 & 129 \\
\hline 2 & 7 & 0.9 & 110.5 \\
\hline 3 & 9 & 1.4 & 144.8 \\
\hline 4 & 10 & 1 & 130 \\
\hline 5 & 12 & 1.2 & 139 \\
\hline 6 & 15 & 1.1 & 137.7 \\
\hline 7 & 16 & 1.2 & 150.4 \\
\hline 8 & 18 & 1.1 & 130 \\
\hline 9 & 19 & 1 & 137 \\
\hline 10 & 20 & 1.08 & 137 \\
\hline 11 & 21 & 1.03 & 138 \\
\hline 12 & 22 & 1.08 & 137 \\
\hline 13 & 23 & 1.6 & 139 \\
\hline 14 & 24 & 0.9 & 131 \\
\hline 15 & 25 & 0.8 & 139 \\
\hline 16 & 26 & 1.2 & 138 \\
\hline 17 & 27 & 1.6 & 141 \\
\hline 18 & 29 & 0.7 & 123 \\
\hline 19 & 31 & 0.9 & 134 \\
\hline 20 & 32 & 0.9 & 130 \\
\hline 21 & 33 & 0.4 & 135 \\
\hline 22 & 34 & 1.1 & 135 \\
\hline 23 & 35 & 1 & 130 \\
\hline 24 & 36 & 0.9 & 108 \\
\hline 25 & 37 & 1.3 & 128 \\
\hline 26 & 38 & 1.08 & 137 \\
\hline 27 & 40 & 1.6 & 140 \\
\hline 28 & 41 & 0.3 & 141 \\
\hline 29 & 48 & 0.8 & 124 \\
\hline \multirow[t]{2}{*}{30} & 50 & 1.3 & 132 \\
\hline & Mean & 1.04233 & 128.88 \\
\hline
\end{tabular}

above table shows mean serum magnesium level is 1.04233 in hypomagnesemic patients and mean s. potassium level was 128.88 and $\mathrm{p}$ value is 0.089204 .

Table -5- Showing Normal Serum Magnesium Level With Serum Sodium Level In Case Of Critically Ill Patients

\begin{tabular}{|l|c|c|c|}
\hline s.no & Case no. & Normomagnesemia & Hyponatremia \\
\hline 1 & 2 & 1.8 & 142 \\
\hline 2 & 3 & 1.8 & 142 \\
\hline 3 & 5 & 1.9 & 137 \\
\hline 4 & 8 & 1.7 & 134 \\
\hline 5 & 11 & 1.8 & 138 \\
\hline 6 & 13 & 1.8 & 136 \\
\hline 7 & 14 & 1.8 & 145 \\
\hline 8 & 28 & 1.8 & 142 \\
\hline 9 & 30 & 1.8 & 127 \\
\hline 10 & 39 & 1.9 & 135 \\
\hline 11 & 42 & 1.9 & 141 \\
\hline 12 & 44 & 1.8 & 137 \\
\hline 13 & 45 & 1.9 & 137 \\
\hline 14 & 47 & 1.8 & 131 \\
\hline 15 & 49 & 1.9 & 138.2 \\
\hline & Mean & 1.82667 & \\
\hline
\end{tabular}


above table shows mean serum magnesium level is 1.82667 in normomagnesemic patients and

Table -6 This Table Showing Low Serum Magnesium Level With Outcome In Case of Critically Ill Patients

\begin{tabular}{|c|c|c|c|}
\hline s.no. & Case no. & Hypomagnesaemia & Outcome(DEATH) \\
\hline 1 & 6 & 0.8 & 1 \\
\hline 2 & 7 & 0.9 & 1 \\
\hline 3 & 9 & 1.4 & 1 \\
\hline 4 & 10 & 1 & 2 \\
\hline 5 & 12 & 1.2 & 2 \\
\hline 6 & 15 & 1.1 & 1 \\
\hline 7 & 16 & 1.2 & 1 \\
\hline 8 & 18 & 1.1 & 2 \\
\hline 9 & 19 & 1 & 1 \\
\hline 10 & 20 & 1.08 & 2 \\
\hline 11 & 21 & 1.03 & 2 \\
\hline 12 & 22 & 1.08 & 1 \\
\hline 13 & 23 & 1.6 & 2 \\
\hline 14 & 24 & 0.9 & 2 \\
\hline 15 & 25 & 0.8 & 2 \\
\hline 16 & 26 & 1.2 & 2 \\
\hline 17 & 27 & 1.6 & 2 \\
\hline 18 & 29 & 0.7 & 2 \\
\hline 19 & 31 & 0.9 & 2 \\
\hline 20 & 32 & 0.9 & 1 \\
\hline 21 & 33 & 0.4 & 1 \\
\hline 22 & 34 & 1.1 & 2 \\
\hline 23 & 35 & 1 & 2 \\
\hline 24 & 36 & 0.9 & 1 \\
\hline 25 & 37 & 1.3 & 1 \\
\hline 26 & 38 & 1.08 & 2 \\
\hline 27 & 40 & 1.6 & 2 \\
\hline 28 & 41 & 0.3 & 2 \\
\hline 29 & 48 & 0.8 & 1 \\
\hline \multirow[t]{2}{*}{30} & 50 & 1.3 & 1 \\
\hline & & MEAN-1.04233 & $43.33 \%$ \\
\hline
\end{tabular}

Above table shows mean serum magnesium level is 1.04233 in hypomagnesemic patients and outcome in form of death is-43.33\%. In above tables on outcome 2 indicate discharge and 1 indicate is death.

\section{Discussion}

Last word about the pathogenesis and treatment of any disease has not been told..everyday some new explanation is put forward to explain the clinical features of disease process, some New regimen treatment is introduced. study of electrolyte disturbances and other biochemical investigations have opened up new chapter in understanding of disease process. until recently magnesium mean s. sodium level was 138.2 and $\mathrm{p}$ value is 0.7499 .

metabolism has been a much neglected subject and it was mainly due to the reason that estimation of this cation was difficult.

Magnesium is mostly located in bone or within the cells. Assessment of magnesium status in either of these compartments in critical illness is impractical. The physician must rely on determination of serum magnesium if a patient has clinically magnesium deficiency symptoms.

Occurrence of magnesium deficiency in critical illness correlates with higher mortality and morbidity. Primary end points of this study were serum magnesium level and outcome in critically ill patients. In our study of 50 critically ill patients, 30 patients were hypomagnesemic, 15 patients were normomagnesemic and 5 patients were hypomagnesemic which signifying remarkable occurrence of hypomagnesemia in critically ill icu patients. Studies carried out throughout the world in critically ill patients have shown varying incidences of magnesium deficiency ranging from $14 \%$ to $66 \% .^{113}$.the causes of magnesium deficiency in critically ill patients are multiple, the major ones being gastrointestinal losses and renal losses. Other risk factors associated with magnesium deficiency are alcoholism and uncontrolled diabetes mellitus. It is important to address the causes of magnesium deficiency while treating hypomagnesemia, to prevent future recurrences.

In our study a relatively higher mortality was observed among patients with low magnesium level compared to normal magnesium level. The relation between hypomagnesemia and mortality varies from study to study. Chernow et al. ${ }^{114}$ (41\%vs13\%), Rubiez et al. $^{109}(46 \%$ vs $25 \%)$, and safavi et al. ${ }^{115}(55 \%$ vs $35 \%)$ had found higher mortality whereas guerin et al. ${ }^{116}(18 \%$ vs $17 \%)$ had found no difference in mortality between hypomagnesemic and normomagnesemic patients in their studies. The higher mortality in low magnesium patients can be explained by increased association of morbidities like more need and 
longer duration of mechanical ventilation, association of diabetes and sepsis, other electrolyte abnormalities like hypokalemia and hyponatremia in compared to patients with normal magnesium levels. The length of ICU stay was longer in hypomagnesemia patients compared to normal magnesium level patients. Soliman et al. ${ }^{110}$ also observed longer duration of ICU stay in patients with low magnesium level. They also found the length of ICU stay as an independent risk factor for hypomagnesemia development.

Hypomagnesemia is known to cause muscle weakness and respiratory failure. This is an important factor causing difficulty in weaning the patients from mechanical ventilation. In the present study we also found that hypomagnesemic patients needed higher mechanical ventilation and also for a longer duration as compared to normal magnesium level patients. C S Limaye et al also observed the same in their study. Safavi et al. ${ }^{115}$ found also the longer duration of mechanical ventilation ( 7.2 vs 4.7 days) in patients with low magnesium level. Fiaccordori et al. ${ }^{117}$ observed that patients with low magnesium were on ventilatory support for more number of days. hypomagnesemia has important role in sepsis, as there is increased release of endothelin and inflammatory cytokines. Harkema et al. ${ }^{118}$ had administered ATP-MgCl2 to the animals models with sepsis and shock in order to restore cellular bioenergetics and observed improved organ function and better survival. Salem et al. ${ }^{119}$ observed that progressive magnesium deficiency and hypomagnesemia are strongly associated with increased mortality in experimental sepsis and magnesium replacement provides significant protection against endotoxin challenge.sepsis is an independent risk factor for developing hypomagnesemia during ICU stay as observed by Soliman et al. In our study we found that incidence of sepsis was more common in patients with low magnesium level compared to normal magnesium level patients.

In our study hypomagnesemia was more common in diabetes patients. CS Limaye et also observed the same in their study. Associated hypomagnesemia in diabetes patients may occur due to increased renal losses accompanies glycosuria. There is strong relationship between hypomagnesemia and insulin resistance ${ }^{120}$. epidemiological studies have shown a high prevalence of hypomagnesemia and lower intracellular magnesium concentrations in diabetes patients. Reduced intracellular magnesium concentrations result in defective tyrosine kinase activity, post-receptorial impairment in insulin action, and worsening of insulin resistance in diabetes patients.

Electrolyte abnormalities are also commonly associated with low magnesium level patients as compared to normal magnesium level patients. In our study we observed increased association of hypokalemia and hyponatremia in patients with low magnesium level as compared to normal magnesium level patients. CS Limaye et al. Also observed increased association of hypokalemia and hypocalcemia in hypomagnesemia patients compared with normal magnesium level patients. Hypokalemia and hypocalcemia seen in hypomagnesemic patient is relatively refractory to potassium and calcium supplementation until the magnesium deficiency is corrected.

Hypermagnesemia is a less frequently incountered problem in ICU patients than hypomagnesemia.In our study it was ten percentage, whereas literature shows a range of 4 to $14 \%{ }^{110,116}$.further and larger study are needed for this. Though the mortality rate was associated significantly with low magnesium level it didn't correlated significantly with low magnesium in this study;further and larger studies are needed to conclude that.

\section{Conclusion}

There was a high prevalence of hypomagnesemia is critically ill medical patient

Hypomagnesaemia was associated with highermortality rate, longer ICU stay, the more frequent need for ventilatory support and with longer duration, and commonly associated with sepsis and diabetes mellitus and other electrolyte 
abnormalities like hypokolamia \& hyponatremia in compared To patients with normal magnesium Sepsis \& organ failure are the strong predominant underlying factor for hypomagnesaemia.

Mortality rate was significantly associated with low magnesium level.

This study gives impact \& significance of serum magnesium monitoring in critically all patients and its value for favorable outcome ,but the need for correction and benefit of magnesium supplement requires further study. 